\title{
Discromías como motivo de consulta; presentación de varios casos
}

\author{
MC. Cubero Sobrados ${ }^{\mathrm{a}}$, D. Agüero Orgaz ${ }^{\mathrm{b}}$, O. Martín Gordo ${ }^{\mathrm{b}}$ \\ aPediatra. CS Orcasitas, Servicio Madrileño de Salud, Área II. Madrid. España. \\ ${ }^{b}$ Médico de Familia. CS Orcasitas, Servicio Madrileño de Salud, Área II. Madrid. España.
}

\begin{abstract}
Resumen
Los trastornos de pigmentación son una causa frecuente de consulta en Atención Primaria, especialmente en pacientes con pieles más pigmentadas. Estos pacientes tienen una mayor predisposición para responder a la presencia de dermatosis y frente a distintas lesiones con alteraciones de la pigmentación, en ocasiones persistentes. Se presentan los casos de un niño de 18 meses de edad y una mujer de 14 años que presentan los hallazgos clínicos característicos de una discromía postinflamatoria, y de una paciente de 13 años con acantosis nigricans.
\end{abstract}

Palabras clave: Discromía. Hiperpigmentación. Hipopigmentación. Acantosis nigricans.

Dyschromia as a cause of consultation; presentation of several case reports

Abstract

Pigmentation disorders are a frequent cause of consultation in primary care, especially in patients with pigmented skins. These patients have a greater predisposition to respond to the presence of dermatosis and to different injuries with alterations of the pigmentation, sometimes persistent. The case of an 18 months old boy, and a 14 years old girl with characteristic clinical findings and a 13 years old girl with acanthosis nigricans are presented.

Key words: Dyschromia. Hyperpigmentation. Hypopigmentation. Acanthosis nigricans.

\section{Introducción}

Las discromías son todas aquellas alteraciones, fisiológicas o patológicas, del color normal de la piel'. Generalmente tienen un curso benigno, pero pueden representar en algunos casos un problema estético ${ }^{1-3}$, que suele ser fre- cuente motivo de consulta en Atención Primaria.

Su tratamiento es difícil, ya que en general el defecto en la pigmentación no es reversible, y en otras ocasiones la mejor opción terapéutica es no hacer nada porque algunas de estas discromías pueden

M. ${ }^{a}$ Carmen Cubero Sobrados, kr_qb@hotmail.com

Los autores declaran no presentar conflictos de intereses en relación con la preparación y publicación de este artículo. 
ir atenuándose espontáneamente con el paso del tiempo. Las alteraciones de la pigmentación cutánea tras la resolución de un proceso inflamatorio cutáneo son una patología que se observa de forma más llamativa en las pieles con fototipos altos $s^{4,5}$.

Su frecuencia real es difícil de precisar, pues son muchas las dermatosis cutáneas que pueden ocasionarlas.

La acantosis nigricans ${ }^{1}$ es una enfermedad rara de la piel, caracterizada por la presencia de hiperqueratosis e hiperpigmentación (lesiones de color gris-parduzco y engrosadas, que dan un aspecto verrugoso y superficie aterciopelada) en los pliegues cutáneos perianales y de las axilas.

\section{Casos clínicos}

1. Niño de 18 meses de edad, que acude a la consulta por presentar una lesión hiperpigmentada en el dorso del pie derecho de aparición hace tres meses (figura 1); previamente ha sufrido una lesión (tipo rozadura) con enrojecimiento por roce de calzado en la zona, sin llegar a producirse ampolla.

2. Paciente de 14 años que acude a la consulta porque no le desaparecen de la pierna las lesiones residuales tras la realización de un tatuaje de henna realizado

Figura 1. Hiperpigmentación residual en dorso de pie izquierdo por roce. 
Figura 2. Hiperpigmentación residual, tras tatuaje de henna, localizada en el dorso de la pierna derecha.

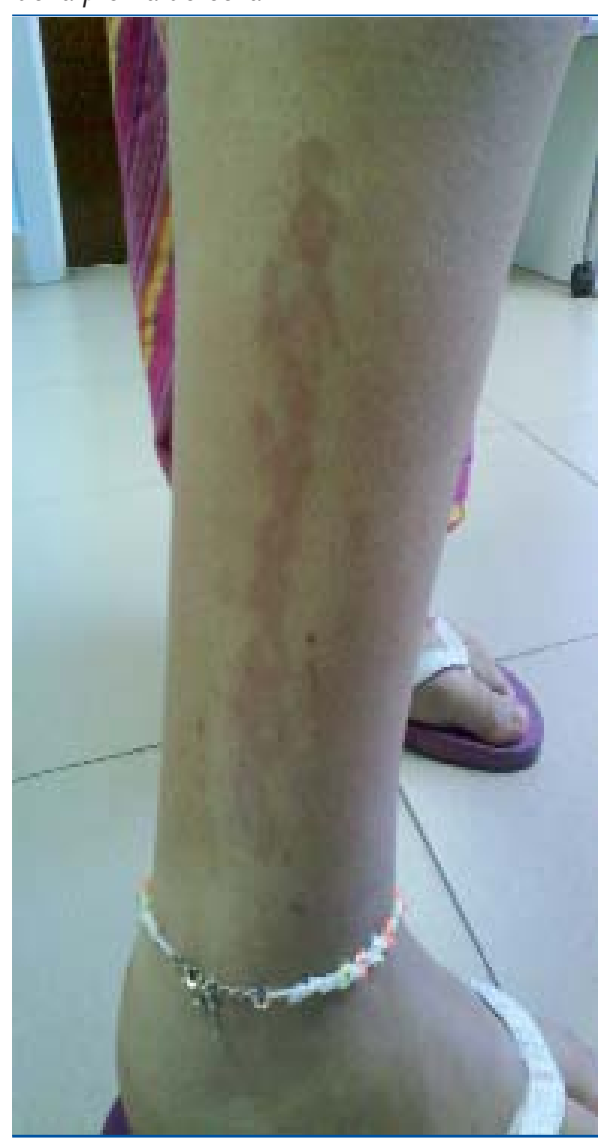

dos meses antes y que le produjo una reacción alérgica con respuesta inflamatoria en la región lateral externa de la pierna derecha (figura 2).

3. Paciente de 13 años que consulta por hiperpigmentación cutánea en la zona del cuello y escote (figura 3); sin antecedentes de interés, salvo presentar sobrepeso (índice de masa corporal $25 \mathrm{~kg} / \mathrm{m}^{2}$ ).

\section{Discusión}

Entre las dermatosis más frecuentes que afectan a los niños y que ocasionan hiper- o hipopigmentación residual se encuentran dermatosis inflamatorias como la dermatitis atópica y la pitiriasis alba, dermatitis de contacto, dermatitis seborreica, lupus discoide y psoriasis, entre otros; infecciones de la piel como el impétigo, la varicela o el herpes; así como las producidas por la aplicación de corticoides tópicos, y son frecuentes las ocasionadas por agresiones físicas a la piel por fricción o rascado, quemaduras y traumatismos ${ }^{1-3}$. Así, está descrita la llamada melanosis por fricción, que se trata de una hiperpigmentación difusa, mal delimitada, de superficie lisa, sin ninguna otra lesión secundaria y asintomática, que aparece en sitios que tienen una base ósea, como las regiones escapular y clavicular.

El diagnóstico está basado fundamentalmente en la historia clínica, en la que resulta especialmente importante insistir en los antecedentes de patologías dermatológicas y tratamientos recibidos, así como en antecedentes traumáticos de todo tipo, ya sean roces, heridas, quemaduras, o exposición solar o a químicos ${ }^{1,2,4}$.

La importancia de las discromías postinflamatorias radica en su prevención, dada la evolución a hiper- o hipopig- 


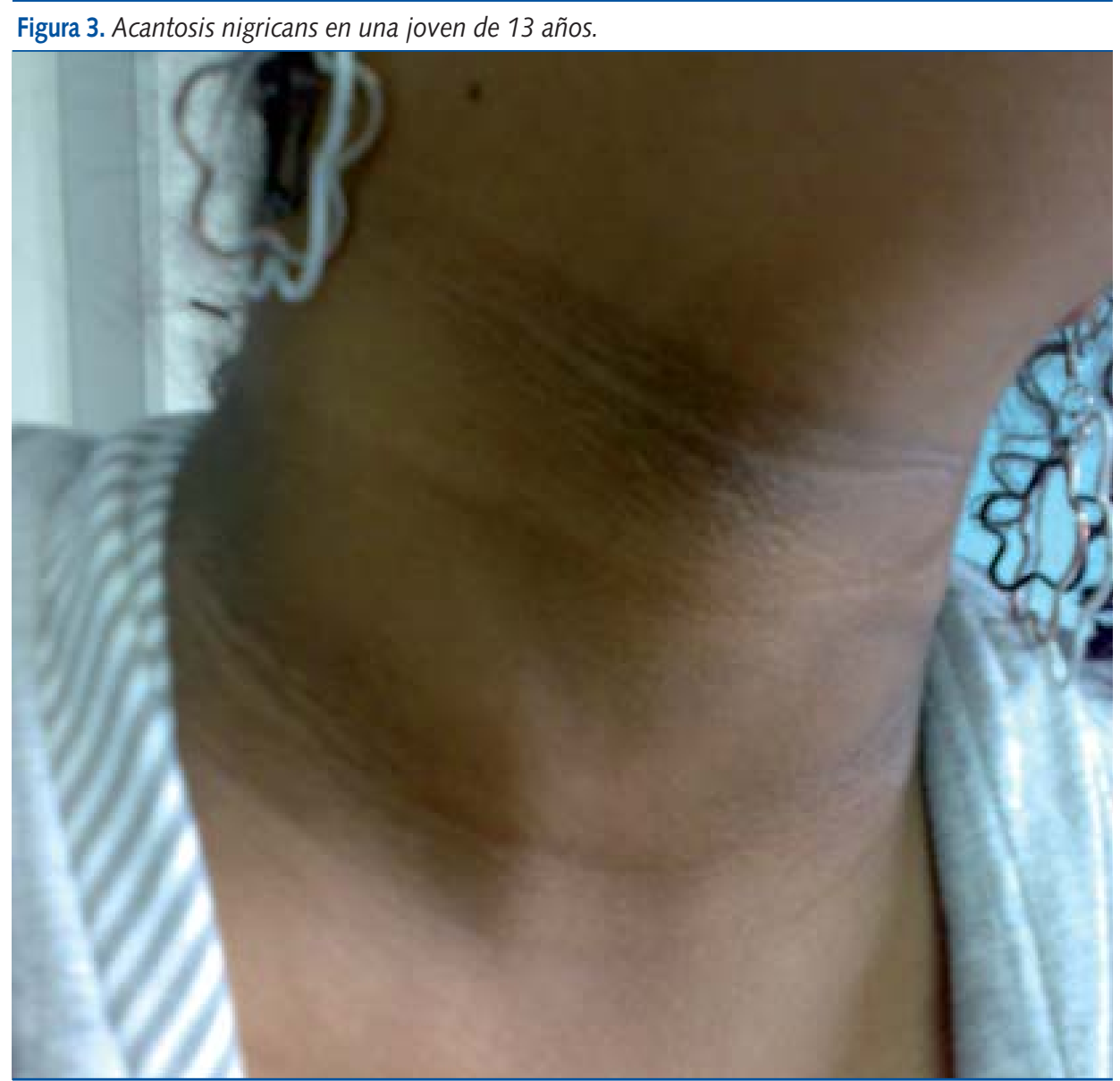

mentación residual marcada en algunos casos, y con consecuencias en ocasiones poco estéticas para el paciente ${ }^{3}$. En aquellos casos en que sea posible habrá que instaurar precozmente el tratamiento de la enfermedad de base para minimizar el consecuente trastorno residual de la pigmentación $n^{1,2}$.

La acantosis nigricans es una hiperpigmentación que se presenta en la diabetes tipo 2, el síndrome metabólico y la obesidad' $^{1}$. Al parecer, la incrementada concentración de insulina activa o estimula ciertos receptores celulares promotores de la proliferación de células de la piel. La consulta en estos casos suele ser por problema estético que causa esta discromía. Se habla de pseudoacantosis nigricans cuando es una forma juvenil benigna asociada a obesidad. 


\section{Bibliografía}

1. Leis Dosil VM, Arránz Sánchez DM, Díaz Díaz RM. Discromías con hiperpigmentación. JANO. 2010;(1759):47-53.

2. Savall López-Reynals R. Valoración de las lesiones cutáneas en la piel negra. FMC. 2007; 14(1):3-12.
3. Llambrich A. Trastornos de la pigmentación. Atlas Dermatología Piel Negra. 2009;11:109-115.

4. Halder RM, Richards GM. Management of dyscromias in ethnic skin. Dermatol Ther. 2004; 17:151-7.

5. Halder RM, Grimes PE, Mc Laurin $C L$, Kress $M A$, KenneyJA. Incidence of common dermatoses in a predominantly black dermatologic practise. Cutis. 1983;32:388-90. 